\title{
Quality of life is improved by endoscopic surgery and fluticasone in nasal polyposis with asthma*
}

\author{
P. Olsson ${ }^{1}$, A. Ehnhage ${ }^{1,2}$, S. Nordin ${ }^{4}$, P. Stjärne ${ }^{1,3}$ for the NAF2S2 * Study Group \\ * Nasal polyposis and Asthma: effects of FESS and FPND; the Stockholm Study \\ 1 Department of Clinical Science, Intervention and Technology, Division of Otorhinolaryngology, Karolinska \\ Institutet, Stockholm, Sweden \\ 2 Nacka Närsjukhus Proxima AB, Stockholm, Sweden \\ 3 Centre for Allergy Research, Karolinska Institutet, Stockholm, Sweden \\ ${ }^{4}$ Department of Psychology, Umeå University, Umeå, Sweden and Department of Psychology, San Diego \\ State University, San Diego, USA
}

\begin{abstract}
SUMMARY
Background: The aim was to investigate the health impact of nasal polyposis with asthma and to study effects of endoscopic sinus surgery (ESS), and addition of fluticasone propionate nasal drops (FPND), on health related quality of life (HRQoL).

Methods: Prospective study of 68 patients with nasal polyposis and asthma. Effects were measured with Study 36-Item Short Form (SF-36). A randomized, double-blind, placebo-controlled 14-weeks phase measuring additive effects of FPND $400 \mu \mathrm{g}$ twice daily (b.i.d.) was included.

Results: HRQoL was significantly decreased in both Physical Component Summary, PCS, and Mental Component Summary, MCS, vs reference population. ESS significantly improved PCS, and MCS after five weeks. We found significant additional benefit of FPND on three domains. The increase in HRQoL with FPND reached reference population levels in all domains, as well as in both PCS and MCS, five weeks after ESS.

Conclusions: FPND $400 \mu \mathrm{g}$ b.i.d. can be added to ESS in order to improve, and to reach population levels of, HRQoL already five weeks post-ESS. Physicians should evaluate HRQoL and consider ESS with nasal steroids early in their treatment of these patients.
\end{abstract}

Key words: fluticasone propionate nasal drops, nasal polyposis, quality of life, randomized controlled trial, endoscopic sinus surgery

\section{INTRODUCTION}

Nasal polyposis, a subgroup of chronic rhinosinusitis, is an inflammatory disease in the nasal and paranasal mucosa of unknown etiology ${ }^{(1)}$. Our definition of nasal polyposis is in accordance with the European guideline definition "chronic rhinosinusitis with nasal polyps" (1). Nasal polyposis is associated with asthma ${ }^{(2,3)}$. Nasal polyposis affects up to four per cent of the Northern European population ${ }^{(4,5)}$ and asthma affects up to ten per cent of that population ${ }^{(6,7)}$. Nasal polyposis, with or without asthma, may significantly decrease patients' health related quality of life (HRQoL), especially vitality scored on the HRQoL instrument Short Form 36 (SF-36) ${ }^{(8-11)}$. The objectives of the management of nasal polyposis are: to reduce or eliminate polyps, open the nasal airway, improve or restore the sense of smell, prevent polyp recurrence and improve patients' Quality of life (QoL) ${ }^{(12,13)}$. Medical treatment, with intranasal corticosteroids and oral corticosteroids, is recommended as first line ${ }^{(1)}$. Clinical studies in patients with nasal polyposis have shown that fluticasone propionate nasal drops (FPND) $400 \mu \mathrm{g}$ twice daily (b.i.d.) has statistically significant and clinically relevant effects on polyp size as well as on nasal congestion ${ }^{(14,15)}$. Surgical treatment, e.g. endoscopic sinus surgery (ESS), has not been sufficiently studied and hence, has been reserved for patients who do not satisfactorily respond to medical treatment (1). Recent data indicate that there is an effect of ESS on the lower airways in patients with nasal polyposis and asthma ${ }^{(16)}$. One of the most extensively used generic HRQoL instruments is the Medical Outcomes Trust Short Form 36-Item Health Survey (SF-36) ${ }^{(17,18)}$. It contains 36 items in eight domains covering both physical and mental health and shows good reproducibility and validity ${ }^{(19)}$. We used the generic SF-36 survey as we aimed to study a population with both asthma and nasal polyposis. Furthermore, we wanted to generate data for comparison with other respiratory and non-respiratory diseases.

No correlation between HRQoL and age, gender, nasal symptoms, CT scan or polyp size has been reported in nasal polyposis ${ }^{(20)}$. The HRQoL profile of patients with nasal polyposis in Sweden has been reported earlier in a study where only about 
$30 \%$ had asthma ${ }^{(21)}$. The health profile in Sweden, the effect of ESS and the combination with intranasal corticosteroid treatment on HRQoL in our patient group, with both nasal polyposis and asthma, has not been sufficiently studied ${ }^{(9,10)}$.

The aim of this study was to investigate the health burden incurred by nasal polyposis with concomitant asthma compared with the Swedish general (reference) population and to study the effects of ESS, and the addition of FPND, on HRQoL in this patient group.

\section{MATERIALS AND METHODS}

Table 1. Mean and standard deviation of the SF-36 variables by treatment group and visit.

\begin{tabular}{lcccc}
\hline Item & Treatment & Visit 2 & Visit 3 & Visit 5 \\
\hline PF & Placebo & $79(20)$ & $80(21)$ & $88(16)^{* *}$ \\
& FPND & $84(16)$ & $86(13)$ & $89(22)^{* *}$ \\
RP & Placebo & $59(40)$ & $64(38)$ & $74(36)$ \\
& & & & $\dagger$ \\
& FPND & $75(33)$ & $77(33)$ & $91(23)^{*}$ \\
BP & Placebo & $68(27)$ & $72(25)$ & $73(20)$ \\
& FPND & $72(26)$ & $76(25)$ & $80(24)$ \\
GH & Placebo & $55(23)$ & $60(21)$ & $67(21)^{* *}$ \\
& FPND & $56(23)$ & $60(22)$ & $71(22)^{* * *}$ \\
& & & & \\
VT & Placebo & $48(26)$ & $49(24)$ & $63(17)^{* * *}$ \\
& & & & $\dagger \dagger$ \\
& FPND & $51(23)$ & $59(21)^{*}$ & $74(17)^{* * *}$ \\
SF & Placebo & $75(27)$ & $74(27$ & $81(21))$ \\
& & & & $\dagger \dagger$ \\
& FPND & $71(24)$ & $81(22)^{*}$ & $93(19)^{* * *}$ \\
RE & Placebo & $63(42)$ & $75(40)$ & $77(37)^{*}$ \\
& FPND & $77(39)$ & $74(38)$ & $89(29)$ \\
MH & Placebo & $71(21)$ & $73(21)$ & $79(14)^{* *}$ \\
& FPND & $75(19)$ & $78(14)$ & $85(13)^{*}$ \\
PCS & Placebo & $44(9)$ & $45(8)$ & $48(8)^{*}$ \\
& FPND & $46(9)$ & $48(7)$ & $50(10)^{* *}$ \\
MCS & Placebo & $42(12)$ & $44(12)$ & $47(9)^{*}$ \\
& & & & $\dagger$ \\
& FPND & $44(12)$ & $46(10)$ & $52(9)^{* *}$ \\
\hline
\end{tabular}

Within group comparison to Visit $\left.\left.\left.2:{ }^{*}\right) \mathrm{p}<0.05^{* *}\right) \mathrm{p}<0.01 * * *\right) \mathrm{p}<0.001$

Between groups comparison: $\dagger$ ) $\mathrm{p}<0.05 \dagger$ ) $\mathrm{p}<0.01$

\section{Patients}

Eighty-two patients, 18 years of age or older (range 19-78 years), with a diagnosis of nasal polyposis and asthma were recruited from the ENT department of the Karolinska University Hospital, Huddinge, Stockholm, Sweden from January 2002 to September 2004. They were required to have bilateral nasal polyps upon endoscopic examination and asthma, diagnosed by history and lung function tests, assessed by a pulmonologist. Among inclusion criteria were age $\geq 18$ years, bilateral nasal polyps and asthma. Exclusion criteria included unfit for general anaesthesia, polypectomy within the last six months, illness or medication that may interfere with the study, and pregnant or lactating women. Asthma treatment was not to be changed during the study. Aspirin sensitivity was not an exclusion criterion, and that specific history was not investigated. No aspirin provocation test was performed. For complete inclusion and exclusion criteria, see Table 2. Medications prohibited during the 4-week pre-study wash-out period were intranasal, ocular, intramuscular, intra-articular, oral, intravenous or rectal corticosteroids, high-potency dermatological corticosteroids, nasal cromones, anti-histamines, hydroxyzine, oral, nasal and ocular decongestants. Oral corticosteroids had a one month wash out period prior to Visit 1 . The same medications were prohibited during the treatment phase, except for nasal or oral decongestants on one occasion of maximum five days. Subjects were withdrawn if there was an asthma exacerbation that had to be treated with oral corticosteroids.

\section{Study design}

This randomized, double-blind, placebo-controlled study of 14 weeks (Visits 1-5) was conducted at the ENT and Pulmonary departments of Karolinska University Hospital as previously described ${ }^{(16)}$. The primary endpoint of this study was a between-groups comparison of the SF-36 domain Vitality (VT) at the end of the double-blind treatment period five weeks after ESS (Visit 5). The study protocol, the patient information and consent form, were reviewed and approved by the local independent ethics committee of Karolinska Institutet (Dnr 234:00) and the Swedish Medical Products Agency (MPA 151:384/01) prior to the enrolment of patients.

Table 2. Inclusion and exclusion criteria.

\begin{tabular}{ll}
\hline Inclusion criteria & Exclusion criteria \\
\hline Age $\geq 18$ years & Unfit for general anesthesia \\
& Polypectomy within last 6 months \\
Bilateral nasal polyps & Illness or medication that may interfere with the study \\
& Idiosyncratic reaction to corticosteroids \\
Asthma & $\begin{array}{l}\text { Prohibited medication within wash out period } \\
\text { Participated in clinical trial within } 30 \text { days }\end{array}$ \\
Capable of recording daily symptom scores in diary & Pregnant or lactating women \\
& Women of child bearing potential not using adequate anti-contraceptive method \\
Capable to comply with dosing regiment & Study personal or patients related to study personal \\
& Patients not to be enrolled more than once
\end{tabular}




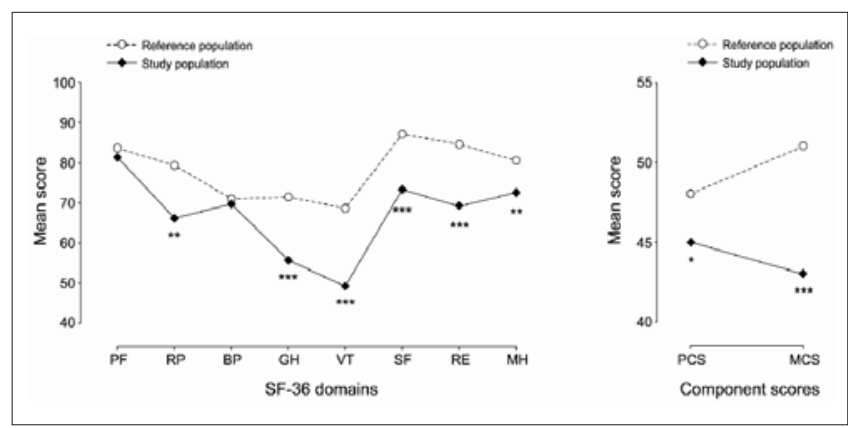

Figure $1 \mathrm{a}$ and $\mathrm{b}$. Baseline SF-36 scores, PCS and MCS. SF-36 scores from the study population and reference population. Six out of eight domains are significantly lower in the study sample. PCS and MCS are significantly lower in the study sample. Data are presented as mean values. Statistically significant differences are indicated; $*$ ) $p<0.05, * *$ ) $\mathrm{p}<0.01$, and $\left.{ }^{* * *}\right) \mathrm{p}<0.001$.

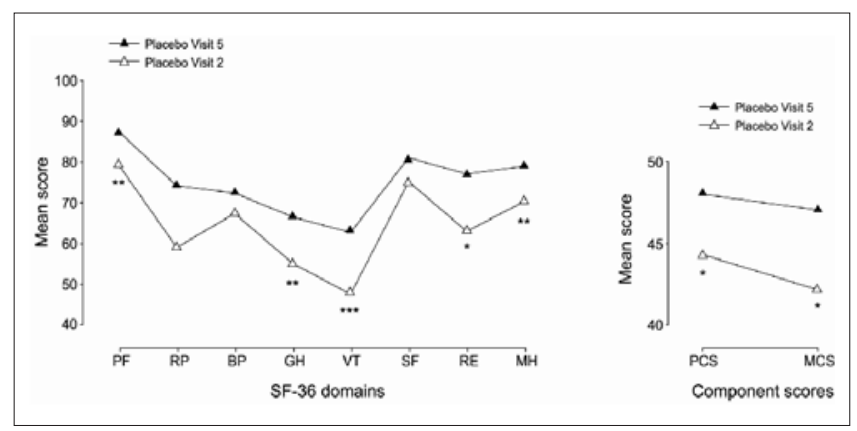

Figures $2 \mathrm{a}$ and b. Effects of ESS (placebo) on SF-36 scores, PCS and MCS. ESS significantly improved five out of eight SF-36 domains as well as Physical Component Summary (PCS) and Mental Component Summary (MCS) after 6 weeks. Data are presented as mean values. Statistically significant changes within the placebo group from Visit 2 are indicated; *) $\left.\mathrm{p}<0.05,{ }^{* *}\right) \mathrm{p}<0.01$, and $\left.{ }^{* * *}\right) \mathrm{p}<0.001$.

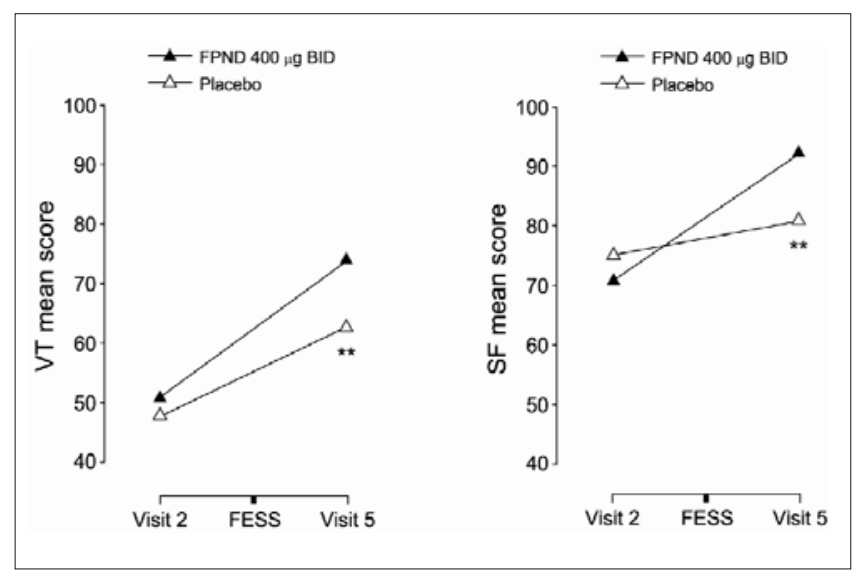

Figures $3 \mathrm{a}$ and $\mathrm{b}$. Effects of ESS and FPND and on a) VT and b) SF domains. FPND $400 \mu$ g b.i.d. gave significant additional benefit versus placebo on three (RP, VT, SF) out of eight domains. Displayed are VT and SF. Data are presented as mean values. Statistically significant differences between the groups at Visit 5 are indicated; $\left.{ }^{* *}\right) \mathrm{p}<0.01$.

\section{Symptoms}

Symptoms from the upper and lower airways were recorded as previously reported ${ }^{(16)}$.

\section{Treatment}

Intranasal treatment

After four weeks wash-out of nasal steroids, at Visit 2, patients were randomized in ratio 1:1 to either placebo or FPND $400 \mu \mathrm{g}$ b.i.d. for 10 weeks as previously described ${ }^{(16)}$.

\section{$\underline{\text { Surgical procedure and follow up }}$}

After four weeks of topical treatment and after clinical examination at Visit 3 patients underwent ESS as previously described ${ }^{(16)}$. Nasal debridement was performed at Visit 4, one week after surgery.

\section{Health related quality of life (SF-36) assessment}

The health survey SF-36 ${ }^{(18)}$ consists of 36 self-administered questions that cover eight health domains: physical functioning $(\mathrm{PF})$, role physical (RP), bodily pain (BP), general health $(\mathrm{GH})$, vitality (VT), social functioning (SF), role emotional (RE) and mental health $(\mathrm{MH})$ that were obtained from the patients at Visit 2, 3 and 5. Scale scores range from 0 to 100 and higher scores indicate better HRQoL. In addition, the physical component summary (PCS) and the mental component summary (MCS) scores were calculated ${ }^{(22)}$ from the eight dimensions of the questionnaire. The Swedish version of SF36 has been translated and validated from the original English language and adapted to the Swedish population ${ }^{(23,24)}$.

\section{Statistical methods}

The reference was an exact sex- and age-matched reference population $(\mathrm{n}=340)$, randomly selected from the Swedish SF36 norm data base $(n=8,930)$. Five reference persons were selected for each patient (quota $=5: 1$ ). The quota was decided from the smallest quota principle, i.e. the smallest number of reference persons corresponding to one patient ${ }^{(25)}$.

According to the Intent-to-treat principle, all randomized subjects in the study population who were given at least one dose of the study treatment and had baseline and post-baseline data were included in the statistical analyses. The SF-36 variables are expressed as means and standard deviations (SD's). Student's t-test was used to compare the SF-36 scores with the Swedish reference population. Changes within groups were analyzed using Wilcoxon signed rank test, and Wilcoxon rank sum test was applied for comparisons of the two treatment groups. For each scale, Cronbach's coefficient was calculated to estimate internal consistency. For study of correlations, Spearman rank-correlation coefficients were calculated. All statistical analyses were performed at a two-sided significance level of 0.05 . The power calculation for determination of sample size was based on clinical symptoms and not on SF-36. The tool for handling and statistical analysis was SPSS 15.0 for Windows (SPSS Inc., Chicago, IL, USA). 


\section{RESULTS}

Sixty-eight patients were randomized (30 to FPND, 38 to placebo) at Visit 2 and 60 patients completed (88\%, 26 with FPND and 34 with placebo) the 14-week randomized, doubleblind, placebo-controlled up to Visit 5. No significant differences were seen at baseline for the SF-36 variables, or for sex (Male 52\% and 67\%) and mean age (51 and 52) between the FPND and placebo/FPND-groups, respectively. Mean polyp score (max 3.0) was 2.2 in the placebo group and 2.3 in the FPND group at baseline.

\section{Quality of life (SF-36) assessment}

At visit 2, HRQoL was significantly decreased with regards to both PCS (45 vs 48, p = 0.049) and MCS ( 43 vs 51, p < 0.001), and six (RP, 66 vs 80, p =0.0048; RE, 69 vs 85, p <0.001; MH, 73 vs $81, \mathrm{p}=0.0015$; VT, 49 vs $69, \mathrm{p}<0.001 ; \mathrm{GH}, 56$ vs 72 , $\mathrm{p}<0.001$; SF, 73 vs $87, \mathrm{p}<0.001$ ) out of eight domains compared with the Swedish reference population (Figure 1 a and b). ESS (placebo group) significantly improved five (PF, $\mathrm{p}=0.002 ; \mathrm{RE}, \mathrm{p}=0.021 ; \mathrm{MH}, \mathrm{p}=0.009 ; \mathrm{VT}, \mathrm{p}<0.001 ; \mathrm{GH}$, $\mathrm{p}=0.005$ ) out of eight domains as well as PCS (44 to 48, $\mathrm{p}=0.027$ ) and MCS (42 to $47, \mathrm{p}=0.021$ ) after approximately five weeks (Visit 5, Figure $2 \mathrm{a}$ and $\mathrm{b}$, Table 1). We found significant benefit of the addition of FPND $400 \mu \mathrm{g}$ b.i.d. versus placebo in three (RP, p = 0.025; VT, p $=0.007$ (Figure $3 \mathrm{a}$ ); SF, $\mathrm{p}=0.002$ (Figure $3 \mathrm{~b}$ )) out of eight domains as well as in MCS ( 52 vs $47, \mathrm{p}=0.013$ ) but not in PCS ( 50 vs $48, \mathrm{p}=0.081$ ). The increase in HRQoL with FPND $400 \mu \mathrm{g}$ b.i.d. reached population levels in all domains (Table 1), as well as in both PCS (50, $\mathrm{p}=0.003)$ and $\operatorname{MCS}(52, \mathrm{p}=0.002)$, approximately five weeks after ESS (Visit 5). Analysis of internal consistencies for all SF36 domains showed Cronbach's coefficients ranging from 0.80 to 0.93 . Values larger than 0.7 are, by convention, considered to be acceptable.

\section{Correlations}

As a post hoc analysis, we studied the population as a whole $(\mathrm{n}=68)$ at Visit 2 to investigate correlations between polyp score, PNIF, olfactory threshold, nasal congestion, sense of smell, PEFR, asthma symptoms and SF-36 variables (including PCS and MCS). The symptoms "shortness of breath" and "nasal congestion" were statistically correlated with PCS ( $\mathrm{R}=-0.52, \mathrm{p}<0.001$ and $\mathrm{R}=-0.44, \mathrm{p}=0.0002$, respectively) and MCS $(\mathrm{R}=-0.28 \mathrm{p}=0.02$ and $\mathrm{R}=-0.37, \mathrm{p}=0.0017$, respectively). The symptom "cough" correlated with PCS $(\mathrm{R}=$ $-0.34, \mathrm{p}=0.005)$, but not with MCS $(\mathrm{R}=-0.10, \mathrm{p}=0.41)$. The symptom "sense of smell" correlated with the SF-36 domain $\mathrm{SF}(\mathrm{R}=0.25, \mathrm{p}=0.04)$ and PNIF correlated with the domain $\mathrm{SF}(\mathrm{R}=-0.26, \mathrm{p}=0.032)$ as well as with MCS $(\mathrm{R}=-0.26$, $\mathrm{p}=0.035)$. Olfactory threshold, polyp score and PEFR were not statistically correlated to QoL.

\section{DISCUSSION}

In this randomized, double-blind, placebo-controlled study of
68 patients, we have found that FPND $400 \mu \mathrm{g}$ b.i.d. can be added to significantly improve clinically relevant positive effects of ESS on HRQoL in patients with nasal polyposis and concomitant asthma. The combination therapy reached population levels of HRQoL already five weeks after surgery.

This study, to our knowledge the largest of its kind, included patients with stable persistent asthma controlled on inhaled corticosteroids, but not requiring oral corticosteroïds, with concomitant nasal polyposis. Despite that the patients' asthma was well controlled with inhaled corticosteroids we noted statistically significant and clinically relevant improvements in five out of eight SF-36 domains, as well as in MCS and PCS, after ESS, without increase in the use of $\beta 2$-agonists.

Given that all but one subject were on inhaled corticosteroids throughout the study and all displayed bronchial hyper responsiveness to histamine in mild to moderate range - indicating good asthma control, the results suggest that ESS has statistically significant positive effects on physical functioning, emotions, mental health, vitality (tiredness, energy level), and general health, according to both the specific and summarized domains of SF-36, already after five weeks. A minimal important difference of 5 scale points has been suggested to be of clinical relevance ${ }^{(26)}$. In this study MCS and all 6 out of 8 domains with statistically significant differences vs the reference population at baseline also had a clinical relevant difference of $\geq 5$ points. This also applies to 6 out of 8 domainimprovements after ESS. Johansson et al. ${ }^{(21)}$ found impairment in only 3 (PF, GH, VT) domains in a group of 44 patients with nasal polyps. The difference in that finding, as well as lack of major impact on mental health compared to our study, might be explained by the fact that their patients were on treatment and that a minority of them were asthmatics (36\%).

Until recently, there has been a lack of randomized study data on nasal polyposis and QoL. Only a few studies have provided information on the effect of treatment of nasal polyposis. No randomized prospective study has, to our knowledge, exclusively studied the nasal polyposis with asthma population from a QoL aspect ${ }^{(27,28)}$. Bousquet and coworkers ${ }^{(29,30)}$ showed that the SF-36 was reliable and valid when used for the assessment of QoL impairment in subjects with moderate asthma. QoL in patients with nasal polyposis associated with asthma was worse than that found in subjects with nasal polyposis without asthma. QoL scores in asthmatic patients without nasal polyposis ${ }^{(29)}$ are better than those of patients with isolated nasal polyposis, suggesting that nasal polyposis impairs QoL to a higher degree than asthma. However, nasal polyposis and asthma seem to have a cumulative negative effect on QoL ${ }^{(8)}$.

Radenne and coworkers ${ }^{(8)}$ investigated the impact of nasal polyposis on QoL in patients, in which the SF-36 questionnaire showed a high internal validity and reliability and that nasal polyposis impaired QoL to a greater degree than perennial allergic rhinitis. In that study, the impairment of QoL was greater 
when nasal polyposis was associated with asthma and pulmonary function was highly correlated to SF-36 scores. We found that the lower airway symptoms "shortness of breath", "cough" and the upper airway symptom "nasal congestion", after washout from upper airway treatment for four weeks, were correlated to generic QoL. This may indicate that these three specific symptoms of nasal polyposis and asthma account for an important part of the effects on QoL in the patient population. We also found a correlation between the symptom "sense of smell" and the SF-36 domain SF, confirming the finding of others that olfactory disorders could alter quality of life ${ }^{(31)}$.

More recently, Alobid et al. ${ }^{(20)}$ demonstrated that QoL was impaired in patients with nasal polyposis compared with the general population. Impairment was observed in all SF-36 domains except in PF. In our patients, impairment compared to the general population was found in all domains except for $\mathrm{PF}$ and BP. We are not surprised of the fact that we did not find an impairment of the domain BP, as we studied nasal polyposis patients with asthma, excluding patients with chronic rhinosinusitis without polyps, which - from clinical experience - are more prone to report pain.

We found significant benefit of the addition of FPND $400 \mu \mathrm{g}$ b.i.d. on perceiving physical limitations, vitality and social functioning as well as on mental health in general. The increase in HRQoL with FPND reached population levels in all domains (Table 1), as well as in both PCS and MCS approximately five weeks after ESS, which is earlier that in other studies that have included patients with nasal polyposis with or without asthma $(8,9,20)$. The additional effect of FPND was not seen when we studied clinical parameters, such as FEV1, PEFR, asthma and nasal symptoms ${ }^{(16)}$. In patients with nasal polyposis, concomitant asthma and atopy had an additional negative impact on QoL scores on RP, BP, VT, and MH ${ }^{(11)}$. Alobid et al. ${ }^{(9)}$ also demonstrated a significant improvement in patients with nasal polyposis on all SF-36 domains after both medical (oral corticosteroids and intranasal steroids) and surgical (ESS followed by 12 months of intranasal steroid) treatment reaching the QoL level of the general population after 6 months.

Nasal polyposis could impair QoL to a higher degree than asthma even in our study, but it is difficult from our results - as all patients had both asthma and nasal polyposis - to assess which disease or symptoms that drive the impact on HRQoL. Furthermore, the question still remains about the possible benefits from endoscopic sinus surgery itself in nasal polyposis patients with concomitant asthma, proven by a randomized controlled study.

In conclusion, this randomized, double-blind, placebo-controlled study shows that HRQoL is significantly impaired in nasal polyposis with asthma. FPND $400 \mu \mathrm{g}$ b.i.d. can be added to improve, and to reach population levels of HRQoL already five weeks post-ESS. We believe that these data indicate that

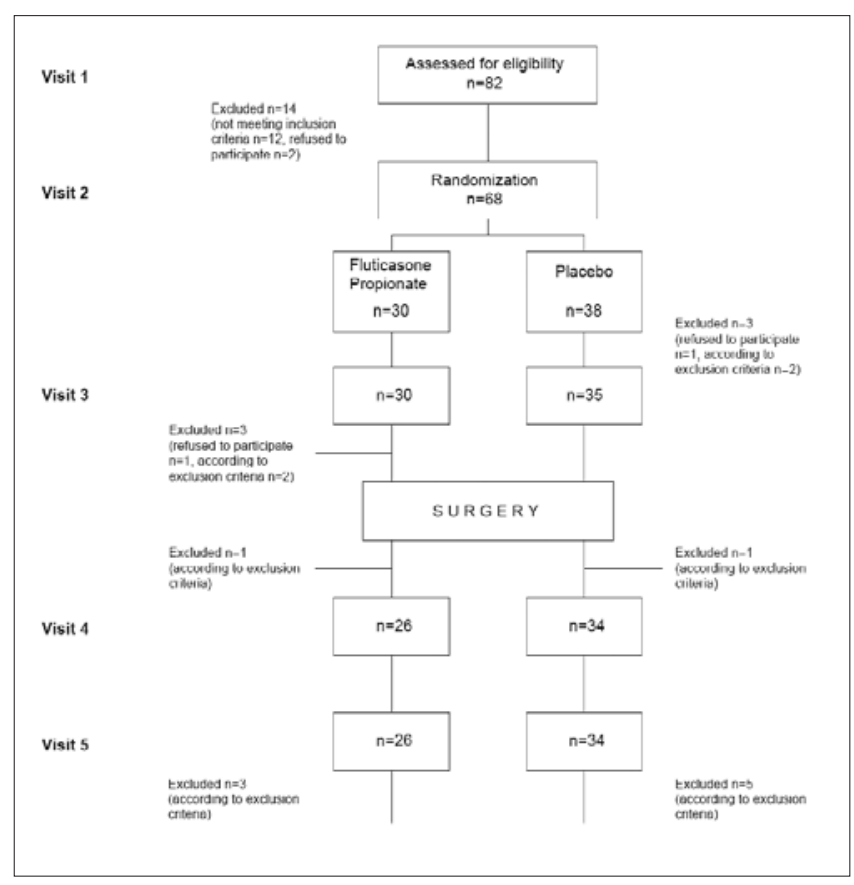

Figure 4. Study flow chart.

physicians should evaluate HRQoL in their assessment of this patient group

\section{ACKNOWLEDGEMENTS}

The authors wish to thank ClinFile AB (Martin Ålenius) for statistical analysis and Johan Hellgren for reviewing. We also thank Vania Sandberg, Agneta Gülich, Ann-Sofie Lantz, Christa Elgerius, Marianne Eduards, Katarina Söderström, Karl-Gustav Kölbäck, Maria Skedinger, Barbro Dahlén, Gert Henriksson, Peter Graf and Karin Toll for their participation in this study.

Financial support for this study was provided by GlaxoSmithKline, the Swedish Association of Otorhinolaryngology, Head and Neck Surgery, the Acta Otolaryngologica Foundation, and the Swedish Asthma and Allergy Association.

\section{POTENTIAL CONFLICTS OF INTEREST}

Dr Olsson joined Schering-Plough AB after the end of this study. Drs Ehnhage and Stjärne have received honoraria from Schering-Plough and GlaxoSmithKline for educational activities. Dr Stjärne has received honoraria for consulting on advisory boards for Schering-Plough, GlaxoSmithKline and Novartis. 


\section{REFERENCES}

1. Fokkens W, Lund V, Mullol J. EP3OS 2007: European position paper on rhinosinusitis and nasal polyps 2007. A summary for otorhinolaryngologists. Rhinology. 2007; 45: 97-101.

2. Samter M, Beers RF, Jr. Intolerance to aspirin. Clinical studies and consideration of its pathogenesis. Ann Int Med. 1968; 68: 975-983.

3. Larsen K. The clinical relationship of nasal polyps to asthma. Allergy Asthma Proc. 1996; 17: 243-249.

4. Hedman J, Kaprio J, Poussa T, et al. Prevalence of asthma, aspirin intolerance, nasal polyposis and chronic obstructive pulmonary disease in a population-based study. Int J Epidemiol. 1999; 28: 717 722 .

5. Johansson L, Åkerlund A, Holmberg K, et al. Prevalence of nasal polyps in adults: the Skovde population-based study. Ann Otol Rhinol Laryngology. 2003; 112: 625-629.

6. Janson C, Chinn S, Jarvis D, et al. Physician-diagnosed asthma and drug utilization in the European Community Respiratory Health Survey. Eur Respir J. 1997; 10: 1795-1802.

7. Larsson ML, Frisk M, Hällström J, et al. Environmental tobacco smoke exposure during childhood is associated with increased prevalence of asthma in adults. Chest. 2001; 120: 711-717.

8. Radenne F, Lamblin C, Vandezande LM, et al. Quality of life in nasal polyposis. J Allergy Clin Immunol. 1999; 104: 79-84.

9. Alobid I, Benitez P, Bernal-Sprekelsen M, et al. Nasal polyposis and its impact on quality of life: comparison between the effects of medical and surgical treatments. Allergy. 2005; 60: 452-458.

10. Alobid I, Bernal-Sprekelsen M, Mullol J. Chronic rhinosinusitis and nasal polyps: the role of generic and specific questionnaires on assessing its impact on patient's quality of life. Allergy. 2008; 63: 1267-1279.

11. Alobid I, Benitez P, Bernal-Sprekelsen M, et al. The impact of asthma and aspirin sensitivity on quality of life of patients with nasal polyposis. Qual Life Res. 2005; 14: 789-793.

12. Mygind N. Advances in the medical treatment of nasal polyps. Allergy. 1999; 54 Suppl 53: 12-16.

13. Tuncer U, Soylu L, Aydogan B, et al. The effectiveness of steroid treatment in nasal polyposis. Auris, Nasus, Larynx. 2003; 30: 263268.

14. Penttilä M, Poulsen P, Hollingworth K, et al. Dose-related efficacy and tolerability of fluticasone propionate nasal drops 400 microg once daily and twice daily in the treatment of bilateral nasal polyposis: a placebo-controlled randomized study in adult patients. Clin Exp Allergy. 2000; 30: 94-102.

15. Holmström M. Clinical performance of fluticasone propionate nasal drops. Allergy. 1999; 54 Suppl 53: 21-25.

16. Ehnhage A, Olsson P, Kölbäck KG, et al. Functional endoscopic sinus surgery improved asthma symptoms as well as PEFR and olfaction in patients with nasal polyposis. Allergy. 2009; 64: 762769.

17. Morley AD, Sharp HR. A review of sinonasal outcome scoring systems - which is best? Clin Otolaryngol. 2006; 31: 103-109.

18. Ware JE, Jr., Sherbourne CD. The MOS 36-item short-form health survey(SF-36). I. Conceptual framework and item selection. Medical care. 199236). I. Conceptual framework and item selection. Medical care. 1992; 3: 473-483.

19. Aaronson NK, Acquadro C, Alonso J, et al. International Quality of Life Assessment (IQOLA) Project. Qual Life Res 1992; 34: 220233.
20. Alobid I, Benitez P, Valero A, et al. The impact of atopy, sinus opacification, and nasal patency on quality of life in patients with severe nasal polyposis. Otolaryngol Head Neck Surg. 2006; 134: 609-612.

21. Johansson L, Brämerson A, Holmberg K, et al. Clinical relevance of nasal polyps in individuals recruited from a general populationbased study. Acta Oto-Laryngologica. 2004; 124: 77-81.

22. Ware JE, Konsinski M Keller SD. SF-36 physical and mental health summary scales: a users manual. Boston, Massachusetts. The Health Institute New England Medical Center, 1994.

23. Sullivan M, Karlsson J. The Swedish SF-36 Health Survey III. Evaluation of criterion-based validity: results from normative population. J Clin Epidemiol. 1998; 51: 1105-1113.

24. Sullivan M, Karlsson J, Ware JE, Jr. The Swedish SF-36 Health Survey--I. Evaluation of data quality, scaling assumptions, reliability and construct validity across general populations in Sweden. Soc Sci Med. 1995; 41: 1349-1358.

25. Sullivan M, Karlsson J, Taft C. SF-36 Hälsoenkät: Svensk Manual och Tolkningsguide, 2:a upplagan. Göteborg: Sahlgrenska Universitetssjukhuset, 2002.

26. McHorney CA, Ware JE, Jr., Raczek AE. The MOS 36-Item Short-Form Health Survey (SF-36): II. Psychometric and clinical tests of validity in measuring physical and mental health constructs. Med Care. 1993 Mar; 31: 247-263.

27. Chester AC, Antisdel JL, Sindwani R. Symptom-specific outcomes of endoscopic sinus surgery: a systematic review. Otolaryngol Head Neck Surg. 2009; 140: 633-639.

28. Hopkins C, Slack R, Lund V, et al. Long-term outcomes from the English national comparative audit of surgery for nasal polyposis and chronic rhinosinusitis. Laryngoscope. 2009; 119: 2459-2465.

29. Bousquet J, Bullinger M, Fayol C, et al. Assessment of quality of life in patients with perennial allergic rhinitis with the French version of the SF-36 Health Status Questionnaire. J Allergy Clin Immunol. 1994; 94: 182-188.

30. Bousquet J, Knani J, Dhivert H, et al. Quality of life in asthma. I. Internal consistency and validity of the SF-36 questionnaire. Am J Resp Crit Care Med. 1994; 149: 371-375.

31. Hummel T, Nordin S. Olfactory disorders and their consequences for quality of life. Acta Oto-Laryngologica. 2005; 125: 116-121.

Petter Olsson, MD, PhD
Department of Clinical Sciences
Intervention and Technology
Division of Otorhinolaryngology
Karolinska Institutet
Karolinska University Hospital Huddinge,
SE-141 86 Stockholm
Sweden

Tel: +46-85-222 1549

Fax: +46-85-222 1501

E-mail: petter.olsson@ki.se 